\title{
Editorial
}

\section{Helping you choose the right journal?}

$\mathrm{W}$ ith new journals being announced every day and many authors being emailed with publishing opportunities how do you decide what is the right place to submit your latest or even your first paper? Natural scientists at the start of their career often rely on the advice of their supervisor about where particular research should be submitted. Recent research in Learned Publishing suggests that if they get to decide themselves they consider a complex matrix of issues which start with journal prestige and impact factor, reflect on the quality of peer review and the rejection rate, look at publication delays and finally consider the Open Access policy. Choosing is not simple.

These days there is also an increasing need to beware of the predatory journals and publishers whose objective is simply to collect a publication fee and who have none of the essential systems in place like peer review, editorial oversight, quality control etc. Beware of unsolicited requests for submissions and, if in doubt, consult the 'blacklists' available at https://beallslist.weebly.com/ or through Cabell's International, which now has a much longer list of over 4000 suspect journals.

So confusing can this choice be that there are now a range of new web services that help authors choose journals but, even here, care is necessary. They differ considerably in the range of journals they include and the accuracy of the data that they hold on these journals. Some charge the author for their services, which can include getting experts to read the draft paper and propose suitable journals for a fee, currently around US\$300. Others are free but that should also suggest caution as their ownership may indicate that they are simply sophisticated advertising systems meant to direct you to a particular publisher. Typical of these are finding services like Elsevier Journal Finder, Springer Journal Suggester and IEEE Publication Recommender, which are each restricted to their own journals. Some finding services are bundled with other purchases so that EndNote Match is funded by Thomson Reuters, tied to the EndNote software many institutions run and linked directly to over 8000 journals in the Web of Science. Another apparently free service, Research Squares Journal Guide, which searches over 46000 journals, is really an editorial consulting firm hoping to sell you editing and translation services.

Of course, you need to publish in a range of journals to ensure that you capture a wide audience for your work. For the paper you wish to submit you have already searched the literature for relevant earlier work giving you a list of journals relevant to what you have just written. And for most disciplines there is a small recognised core group of journals that publish most of the best research, a point substantiated by Eugene Garfield over twenty years ago. But what then of multidisciplinary journals, like this one, whose role is to focus on the region and not the science discipline, and to encourage cross-disciplinary interests?

If your paper is, for instance, on bird diet then it could clearly interest marine biologists as well as ornithologists. If it is on climate then biologists, glaciologists, even chemists could be interested as well as meteorologists. And even at a more specific level a more general range of interests could value your material; climatologists look at sediments for proxies of climate change, toxicologists need chemists to account for the presence of organic pollutants, and so on.

Think carefully who would be able to use your work most effectively and how that audience can most easily be reached. Choosing a journal is not just about citations but, like Antarctic science, a many faceted problem with alternative solutions!

D.W.H. WALTON 